\title{
1 Next generation sequencing to investigate genomic diversity in Caryophyllales
}

2 Boas Pucker ${ }^{1,2^{*}}$, Tao Feng ${ }^{1,3}$, Samuel F. Brockington ${ }^{1,2}$

31 Evolution and Diversity, Plant Sciences, University of Cambridge, Cambridge, United Kingdom

42 Genetics and Genomics of Plants, CeBiTec \& Faculty of Biology, Bielefeld University, Bielefeld,

5 Germany

63 Wuhan Botanical Garden, Chinese Academy of Sciences, Wuhan, China

$7 \quad$ * corresponding author: Boas Pucker, bpucker@cebitec.uni-bielefeld.de

9 BP: bpucker@cebitec.uni-bielefeld.de, ORCID: 0000-0002-3321-7471

10 TF: fengtao@wbgcas.cn, ORCID: 0000-0002-0489-2021

11 SFB: sb771@cam.ac.uk, ORCID: 0000-0003-1216-219X

Key words: whole genome sequencing, genome assembly, anthocyanin, betalain, Kewa caespitosa, Macarthuria australis, Pharnaceum exiguum, Caryophyllales

\section{Abstract}

Caryophyllales are a highly diverse and large order of plants with a global distribution. While some species are important crops like Beta vulgaris, many others can survive under extreme conditions. This order is well known for the complex pigment evolution, because the pigments anthocyanins and betalains occur with mutual exclusion in species of the Caryophyllales. Here we report about genome assemblies of Kewa caespitosa (Kewaceae), Macarthuria australis (Macarthuriaceae), and Pharnaceum exiguum (Molluginaceae) which are representing different taxonomic groups in the 


\section{Introduction}

Caryophyllales form one of the largest flowering plant order and are recognized for their outstanding ability to colonise extreme environments. Examples are the evolution of Cactaceae in deserts, extremely fast radiation [1-3] e.g. in arid-adapted Aizoaceae and in carnivorous species in nitrogenpoor conditions. Caryophyllales harbor the greatest concentration of halophytic plant species and display repeated shifts to alpine and arctic habitats in Caryophyllaceae and Montiaceae. Due to these extreme environments, species exhibit many adaptations [2-4] such as specialized betalain pigments to protect photosystems in high salt and high light conditions [5]. There are several examples for repeated evolution in the Caryophyllales e.g. leaf and stem succulence for water storage, various mechanisms for salt tolerance, arid-adapted $C_{4}$ and CAM photosynthesis [4], and insect trapping mechanisms to acquire nitrogen [6].

In addition, to their fascinating trait evolution, the Caryophyllales are well known for important crops and horticultural species like sugar beet, quinoa and spinach. Most prominent is the genome sequence of Beta vulgaris [7] which was often used as a reference for studies within Caryophyllales [7-10]. In addition, genomes of Spinacia oleracea [7,11], Dianthus caryophyllus [12], Amaranthus hypochondriacus [13], and Chenopodium quinoa [14] were sequenced. Besides Carnegiea gigantea and several other cacti [15], recent genome sequencing projects were focused on crops due to their economical relevance. However, genome sequences of other species within the Caryophyllales, are needed to provide insights into unusual patterns of trait evolution.

The evolution of pigmentation is known to be complex within the Caryophyllales [8] with a single origin of betalain and at least three reversals to anthocyanin pigmentation. The biosynthetic pathways for betalain and anthocyanin pigmentation are both well characterized. While previous studies have demonstrated that the genes essential for anthocyanin synthesis persists in betalain pigmented taxa $[16,17]$, the fate of the betalain pathway in the multiple reversals to anthocyanin pigmentations is unknown. Here, we sequenced three species from different families to contribute to the genomic knowledge about Caryophyllales: Kewa caespitosa (Kewaceae), Macarthuria australis (Macarthuriaceae), and Pharnaceum exiguum (Molluginaceae) were selected as representatives of anthocyanic lineages within the predominantly betalain pigmented Caryophyllales. $K$. caespitosa and P. exiguum are examples of putative reversals from betalain pigmentation to anthocyanic pigmentation, while Macarthuria is a lineage that diverged before the inferred origin of betalain pigmentation [8].

Several transcript sequences of the three plants investigated here were assembled as part of the $1 \mathrm{KP}$ project [18]. Since the sampling for this transcriptome project was restricted to leaf tissue, available sequences are limited to genes expressed there. Here we report three draft genome sequences to 
complement the available gene set and to enable analysis of untranscribed sequences like promoters, regulatory elements, pseudogenes, and transposable elements.

\section{Material \& Methods}

\section{Plant material}

The seeds of Kewa caespitosa (Friedrich) Christenh., Marcarthuria australis Hügel ex Endl., and Pharnaceum exiguum Adamson were obtained from Millennium Seed Bank (London, UK) and were germinated at the Cambridge University Botanic Garden. The plants were grown in controlled glasshouse under conditions: long-day ( $16 \mathrm{~h}$ light and $8 \mathrm{~h}$ dark), $20^{\circ} \mathrm{C}, 60 \%$ humidity. About $100 \mathrm{mg}$ fresh young shoots were collected and immediately frozen in liquid nitrogen. Tissue was ground in liquid nitrogen using a mortar and pestle. DNA was extracted using the QIAGEN DNeasy Plant Mini Kit (Hilden, Germany) and RNA was removed by the QIAGEN DNase-Free RNase Set. DNA quantity and quality were assessed by Nanodrop (Thermofisher Scientific, Waltham, MA, USA) and agarose gel electrophoresis. DNA samples were sent to BGI Technology (Hongkong) for library construction and Illumina sequencing.

\section{Sequencing}

Libraries of $K$. caespitosa, $M$. australis, and P. exiguum were sequenced on an Illumina HiSeq X-Ten generating 2x150nt reads (AdditionalFile 1). Trimmomatic v0.36 [19] was applied for adapter removal and quality trimming as described previously [20]. Due to remaining adapter sequences, the last 10 bases of each read were clipped. FastQC [21] was applied to check the quality of the reads.

\section{Genome size estimation}

The size of all three investigated genomes was estimated based on $k$-mer frequencies in the sequencing reads. Jellyfish v2 [22] was applied for the construction of a k-mer table with parameters described by [23]. The derived histogram was further analyzed by GenomeScope [23] to predict a genome size. This process was repeated for all odd k-mer sizes between 17 and 25 (AdditionalFile 2). Finally, an average value was selected from all successful analyses. 
92

93

94

95

96

97

98

99

100

101

102

103

104

105

106

107

108

109

110

111

112

113

114

115

116

117

118

119

120

121

122

123

124

\section{Genome assembly}

The performance of different assemblers on the data sets was tested (AdditionalFile 3, AdditionalFile 4, AdditionalFile 5). While CLC Genomics Workbench performed best for the M. australis assembly, SOAPdenovo2 [24] showed the best results for $K$. caespitosa and P. exiguum and was therefore selected for the final assemblies. To optimize the assemblies, different k-mer sizes were tested as this parameter can best be adjusted empirically [25]. First, k-mer sizes from 67 to 127 in steps of 10 were evaluated, while most parameters remained on default values (AdditionalFile 6). Second, assemblies with k-mer sizes around the best value of the first round were tested. In addition, different insert sizes were evaluated without substantial effect on the assembly quality. In accordance with good practice, assembled sequences shorter than $500 \mathrm{bp}$ were discarded prior to downstream analyses. Custom Python scripts $[20,26]$ were deployed for assembly evaluation based on simple statistics (e.g. N50, N90, assembly size, number of contigs), number of genes predicted by AUGUSTUS v3.2 [27] ab initio, average size of predicted genes, and number of complete BUSCOs [28]. Scripts are available on github: https://github.com/bpucker/GenomeAssemblies2018.

BWA-MEM v0.7 [29] was used with the $-m$ flag to map all sequencing reads back against the assembly. REAPR v1.0.18 [30] was applied on the selected assemblies to identify putative assembly errors through inspection of paired-end mappings and to break sequences at those points.

The resulting assemblies were further polished by removal of non-plant sequences. First, all assembled sequences were subjected to a BLASTn [31] against the sugar beet reference genome sequence RefBeet v1.5 [7,32] and the genome sequences of Chenopodium quinoa [14], Carnegiea gigantea [15], Amaranthus hypochondriacus [13], and Dianthus caryophyllus [12]. Hits below the evalue threshold of $10^{-10}$ were considered to be of plant origin. Second, all sequences without hits in this first round were subjected to a BLASTn search against the non-redundant nucleotide database nt. Sequences with strong hits against bacterial and fungal sequences were removed as previously described [20,26]. BLASTn against the B. vulgaris plastome (KR230391.1, [33]) and chondrome (BA000009.3, [34]) sequences was performed to identify and remove sequences from these organelle subgenomes.

\section{Assembly quality assessment}

Mapping of sequencing reads against the assembly and processing with REAPR [30] was the first quality control step. RNA-Seq reads (AdditionalFile 7) were mapped against the assemblies to assess completeness of the gene space and to validate the assembly with an independent data set. STAR v2.5.1b [35] was used for the RNA-Seq read mapping as previously described [26]. 


\section{Genome annotation}

127 RepeatMasker [36] was applied using crossmatch [37] to identify and mask repetitive regions prior to gene prediction. Masking was performed in sensitive mode (-s) without screening for bacterial IS elements (-no_is) and skipping interspersed repeats (-noint). Repeat sequences of the Caryophyllales (-species caryophyllales) were used and the GC content was calculated per sequence (-gccalc). Protein coding sequences of transcriptome assemblies (AdditionalFile 7) were mapped to the respective genome assembly via BLAT [38] to generate hints for the gene prediction process as previously described [39]. BUSCO v3 [28] was deployed to optimize species-specific parameter sets for all three species based on the sugar beet parameter set [40]. AUGUSTUS v.3.2.2 [27] was applied to incorporate all available hints with previously described parameter settings to optimize the prediction of non-canonical splice sites [39]. Different combinations of hints and parameters were evaluated to achieve an optimal annotation of all three assemblies. A customized Python script was deployed to remove all genes with premature termination codons in their CDS or spanning positions with ambiguous bases. Representative transcripts and peptides per locus were identified based on maximization of the encoded peptide length. INFERNAL (cmscan) [41] was used for the prediction of non-coding RNAs based on models from Rfam13 [42].

142 Functional annotation was transferred from Arabidopsis thaliana (Araport11) [43] via reciprocal best 143 BLAST hits as previously described [26]. In addition, GO terms were assigned to protein coding genes through an InterProScan5 [44]-based pipeline [26].

\section{Comparison between transcriptome and genome assembly}

147 The assembled genome sequences were compared against previously published transcriptome assemblies (AdditionalFile 7) in a reciprocal way to assess completeness and differences. BLAT [38] was used to align protein coding sequences against each other. This comparison was limited to the protein coding sequences to avoid biases due to UTR sequences, which are in general less reliably predicted or assembled, respectively [39]. The initial alignments were filtered via filterPSL.pl [45]

152 based on recommended criteria for gene prediction hint generation to remove spurious hits and to reduce the set to the best hit per locus e.g. caused by multiple splice variants. 
157

158

159

160

161

162

163

\begin{tabular}{|c|c|c|c|}
\hline Accession & $\begin{array}{l}\text { Kewa caespitose } \\
\text { GCA_900322205 }\end{array}$ & $\begin{array}{l}\text { Macarthuria australis } \\
\text { GCA_900322265 }\end{array}$ & $\begin{array}{l}\text { Pharnaceum exiguum } \\
\text { GCA_900322385 }\end{array}$ \\
\hline $\begin{array}{l}\text { Estimated genome } \\
\text { size [Mbp] }\end{array}$ & 623 & 497.5 & 265 \\
\hline Sequencing coverage & $111 x$ & $251 x$ & $206 x$ \\
\hline Assembly size $(-N)$ & $531,205,354$ & $525,292,167$ & $254,526,612$ \\
\hline Number of sequence & 55,159 & 271,872 & 16,641 \\
\hline N50 & 28,527 & 2,804 & 56,812 \\
\hline Max. sequence length & 340,297 & 211,626 & 514,701 \\
\hline GC content & $38.1 \%$ & $36.6 \%$ & $37.4 \%$ \\
\hline Complete BUSCOs & $83.6 \%$ & $44.4 \%$ & $84.3 \%$ \\
\hline \multirow[t]{2}{*}{ Assembler } & SOAPdenovo2 & Genomics & SOAPdenovo2 \\
\hline & & Workbench v9 & \\
\hline k-mer size & 79 & Automatic & 117 \\
\hline
\end{tabular}

\section{$\underline{\text { Results }}$}

\section{Genome size estimation and genome sequence assembly}

Prior to the de novo genome assembly, the genome sizes of Kewa caespitosa, Macarthuria australis, and Pharnaceum exiguum were estimated from the sequencing reads (Table 1, AdditionalFile 1). The estimated genome sizes range from $265 \mathrm{Mbp}$ ( $P$. exiguum) to $623 \mathrm{Mbp}$ ( $M$. caespitosa). Based on these genome sizes, the sequencing coverage ranges from $111 x$ ( $K$. caespitosa) to $251 x$ (M. australis).

Different assembly tools and parameters were evaluated to optimize the assembly process (AdditionalFile 3, AdditionalFile 4, AdditionalFile 5). Sizes of the final assemblies ranged from $254.5 \mathrm{Mbp}$ (P. exiguum) to $531 \mathrm{Mbp}$ ( $K$. caespitosa) (Table 1, AdditionalFile 8). The best continuity was achieved for $P$. exiguum with an N50 of $57 \mathrm{kbp}$.

Table 1: Genome size estimation and de novo assembly statistics.
168

169

170

171

172

173

174

\section{Assembly validation}

The mapping of sequencing reads against the assembled sequences resulted in mating rates of $99.5 \%$ (K. caespitosa), 98\% (M. australis), and 94.8\% (P. exiguum). REAPR identified between 1390 ( $P$. exiguum) and 16181 (M. australis) FCD errors which were corrected by breaking assembled sequences. The mapping of RNA-Seq reads to the polished assembly resulted in mapping rates of 
$17553.9 \%$ (K. caespitosa) and 43.1\% (M. australis), respectively, when only considering uniquely mapped 176 reads. Quality assessment via BUSCO revealed 83.6\% (K. caespitosa), $44.4 \%$ (M. australis), and 84.3\%

177 ( $P$. exiguum) complete benchmarking universal single copy ortholog genes ( $n=1440)$. In addition, $1786.5 \%$ (K. caespitosa), $21.7 \%$ (M. australis), and 4.0\% (P. exiguum) fragmented BUSCOs as well as 9.9\% 179 (K. caespitosa), 33.9\% (M. australis), and $11.7 \%$ (P. exiguum) missing BUSCOs were identified. The 180 proportion of duplicated BUSCOs ranges from 1.5\% (K. caespitosa) to $2.1 \%$ (P. exiguum). The number 181 of duplicated BUSCOs was high in M. australis (11.8\%) compared to both other genome assemblies 182 (1.5\% and $2.1 \%$, respectively).

\section{Genome annotation}

After intensive optimization (AdditionalFile 9), the polished structural annotation contains between 26,155 (P. exiguum) and 80,236 ( $M$. australis) protein encoding genes per genome (Table 2). The average number of exons per genes ranged from 2.9 (M. australis) to 6.6 ( $K$. caespitosa). Predicted peptide sequence lengths vary between 241 (M. australis) and 447 (K. caespitosa) amino acids. High numbers of recovered BUSCO genes support the assembly quality (Fig. 1). Functional annotations were assigned to between $50 \%$ ( $K$. caespitosa) and $70 \%$ ( $P$. exiguum) of the predicted genes per species. These assemblies revealed between 598 (P. exiguum) and 1604 (M. australis) putative rRNA, 821 ( $K$. caespitosa) to 1492 ( $M$. australis) tRNA genes, and additional non-protein-coding RNA genes (Table 2).

Fig. 1. Assembly completeness.

Assembly completeness was assessed based on the proportion of complete, fragmented, and missing BUSCOs.

197

Table 2: Assembly annotation statistics. These data sets are available at (Macarthuria australis), and https://doi.org/10.4119/unibi/2941045 (Pharnaceum exiguum).

\begin{tabular}{llll}
\hline & Kewa caespitosa & Macarthuria australis & Pharnaceum exiguum \\
\hline Final gene number & 50661 & 80236 & 26,155 \\
Functional annotation assigned & $25,058(49.46 \%)$ & $50,536(62.98 \%)$ & $18,372(70.24 \%)$ \\
Average gene lengths [bp] & 5494 & 1936 & 5090 \\
Average mRNA length [bp] & 2143 & 1018 & 2154 \\
Average peptide length [aa] & 447 & 241 & 435 \\
\hline
\end{tabular}




\begin{tabular}{llll}
\hline RBHs vs. BeetSet2 & 9,968 & 10,568 & 10,045 \\
Average number of exons per & 6.6 & 2.9 & 6 \\
gene & & & 1260 \\
Number of predicted tRNAs & 821 & 1491 & 598 \\
Number of predicted rRNAs & 720 & 1604 &
\end{tabular}

201

202

Comparison between transcriptome and genome assemblies

204

205

206

207

208

209

210

211

212

213

214

215

216

217

218

219

220

221

222

223

224

225

226

227

Previously released transcriptome assemblies were compared to the genome assemblies to assess completeness and to identify differences. In total 44,169 of 65,062 (67.9\%) coding sequences of the $K$. caespitose transcriptome assembly were recovered in the corresponding genome assembly. This recovery rate is lower for both $M$. australis assemblies, where only 27,894 of 58,953 (47.3\%) coding sequences were detected in the genome assembly. The highest rate was observed for $P$. exiguum, where 37,318 of $42,850(87.1 \%)$ coding sequences were found in the genome assembly. When screening the transcriptome assemblies for transcript sequences predicted based on the genome sequences, the recovery rate was lower (Fig. 2). The number of predicted representative coding sequences with best hits against the transcriptome assembly ranged from $16.3 \%$ in $K$. caespitosa to $29.7 \%$ in $P$. exiguum thus leaving most predicted coding sequences without a good full length hit in the transcriptome assemblies.

Fig. 2. Recovery of sequences between transcriptome and genome assemblies.

The figure displays the percentage of sequences present in one assembly that are recovered or missing in the other assembly type.

\section{Discussion}

An almost perfect match between the predicted genome size and the final assembly size was observed for $P$. exiguum. When taking gaps within scaffolds into account the $K$. caespitosa assembly size reached the estimated genome size. High heterozygosity could be one explanation for the assembly size exceeding the estimated haploid genome size of $M$. australis. The two independent genome size estimations for $M$. australis based on different read data sets indicate almost perfect reproducibility of this method. Although centromeric regions and other low complexity regions were 
228 probably underestimated in the genome size estimation as well as in the assembly process, this 229 agreement between estimated genome size and final assembly size indicates a high assembly quality.

230 The continuity of the $P$. exiguum assembly is comparable to the assembly continuity of Dianthus 231 caryophyllus [12] with a scaffold N50 of $60.7 \mathrm{~kb}$. Additional quality indicators are the high proportion 232 of detected BUSCOs in the final assemblies as well as the high mapping rate of reads against the 233 assemblies. The percentage of complete BUSCOs is in the same range as the value of the 234 D. caryophyllales genome assembly which revealed $88.9 \%$ complete BUSCOs based on our BUSCO 235 settings. We demonstrate a cost-effective generation of draft genome assemblies of three different 236 plant species. Investing into more paired-end sequencing based on Illumina technology would not 237 substantially increase the continuity of the presented assemblies. This was revealed by initial 238 assemblies for $M$. australis performed with less than half of all generated sequencing reads. Although 239 the total assembly size increased when doubling the amount of incorporated sequencing reads, the 240 continuity is still relatively low. No direct correlation between the sequencing depth and the assembly quality was observed in this study. Genome properties seem to be more influential than the amount of sequencing data. Even very deep sequencing with short reads in previous studies $[12,20]$ was unable to compete with the potential of long reads in genome assembly projects $[13,14]$. No major breakthroughs were achieved in the development of publicly available short read assemblers during the last years partly due to the availability of long reads which made it less interesting.

The number of predicted genes in $P$. exiguum is in the range expected for most plants $[46,47]$. While the predicted gene numbers for $K$. caespitosa and $M$. australis are much higher than that for P. exiguum, they are only slightly exceeding the number of genes predicted for other plants $[46,47]$. Nevertheless, the assembly continuity and the heterozygosity of $M$. australis are probably the most important factors for the artificially high number of predicted genes. The high percentage of duplicated BUSCOs (11.8\%) indicates the presence of both alleles for several genes. As the average gene length in $M$. australis is shorter than in both other assemblies, some gene model predictions might be too short. This gene prediction could be improved by an increase in assembly continuity.

There is a substantial difference between the transcriptome sequences and the predicted transcripts of the genome assembly. The presence of alternative transcripts and fragmented transcripts in the transcriptome assemblies are one explanation why not all transcripts were assigned to a genomic locus. Another explanation is the intraspecific variation, as the genome and transcriptome assemblies were generated using different individuals. Some transcripts probably represent genes which are not properly resolved in the genome assemblies. This is especially the case for M. australis. 
263

differences. The complete BUSCO percentage of the $P$. exiguum genome assembly even exceeds the value assigned to the corresponding transcriptome assembly. Although BUSCOs are selected in a robust way, it is likely that some of these genes are not present in the genomes investigated here, since B. vulgaris is the closest relative with an almost completely sequenced genome [7]. Our genome assemblies provide additional sequences of genes which are not expressed in the tissues sampled for the generation of the transcriptome assembly. In addition, coding sequences might be complete in the genome assemblies, while low expression caused a fragmented assembly based on RNA-Seq reads. This explains why only a small fraction of the predicted coding sequences of the genome assemblies was mapped to the coding sequences derived from the corresponding transcriptome assembly.

The availability of assembled sequences as well as large sequencing read data sets enables the investigation of repeats e.g. transposable elements across a large phylogenetic distance within the Caryophyllales. It also allows the extension of genome-wide analysis, such as gene family investigations from B. vulgaris to more representatives across Caryophyllales. As all three species produce anthocyanins, we provide the basis to study the underlying biosynthetic genes. Due to the huge evolutionary distance to other anthocyanin producing species, the availability of these sequences could facilitate the identification of common and unique features of the involved enzymes.

\section{Author contribution}

TF isolated DNA. BP and TF performed data processing, assembly, and annotation. BP, TF, and SFB interpreted the results. BP wrote the initial draft. All authors read and approved the final version of this manuscript.

\section{Acknowledgements}

We thank the CeBiTec Bioinformatic Resource Facility team for great technical support.

\section{References}

1. Brockington SF, Walker RH, Glover BJ, Soltis PS, Soltis DE. Complex pigment evolution in the Caryophyllales. New Phytol. 2011;190: 854-864. doi:10.1111/j.1469-8137.2011.03687.x 
2. Yang Y, Moore MJ, Brockington SF, Soltis DE, Wong GK-S, Carpenter EJ, et al. Dissecting Molecular Evolution in the Highly Diverse Plant Clade Caryophyllales Using Transcriptome Sequencing. Mol Biol Evol. 2015;32: 2001-2014. doi:10.1093/molbev/msv081

3. Smith SA, Brown JW, Yang Y, Bruenn R, Drummond CP, Brockington SF, et al. Disparity, diversity, and duplications in the Caryophyllales. New Phytol. 2018;217: 836-854. doi:10.1111/nph.14772

4. Kadereit G, Ackerly D, Pirie MD. A broader model for C4 photosynthesis evolution in plants inferred from the goosefoot family (Chenopodiaceae s.s.). Proc R Soc B Biol Sci. 2012;279: 3304-3311. doi:10.1098/rspb.2012.0440

5. Jain G, Schwinn KE, Gould KS. Betalain induction by I-DOPA application confers photoprotection to saline-exposed leaves of Disphyma australe. New Phytol. 2015;207: 1075-1083. doi:10.1111/nph.13409

6. Thorogood CJ, Bauer U, Hiscock SJ. Convergent and divergent evolution in carnivorous pitcher plant traps. New Phytol. 2018;217: 1035-1041. doi:10.1111/nph.14879

7. Dohm JC, Minoche AE, Holtgräwe D, Capella-Gutiérrez S, Zakrzewski F, Tafer H, et al. The genome of the recently domesticated crop plant sugar beet (Beta vulgaris). Nature. 2014;505: 546-549. doi:10.1038/nature12817

8. Brockington SF, Yang Y, Gandia-Herrero F, Covshoff S, Hibberd JM, Sage RF, et al. Lineagespecific gene radiations underlie the evolution of novel betalain pigmentation in Caryophyllales. New Phytol. 2015;207: 1170-1180. doi:10.1111/nph.13441

9. Stevanato $P$, Trebbi $D$, Saccomani M. Single nucleotide polymorphism markers linked to root elongation rate in sugar beet. Biol Plant. 2017;61: 48-54. doi:10.1007/s10535-016-0643-1

10. Kong W, Yang S, Wang Y, Bendahmane M, Fu X. Genome-wide identification and characterization of aquaporin gene family in Beta vulgaris. PeerJ. 2017;5. doi:10.7717/peerj. 3747

11. Xu C, Jiao C, Sun H, Cai X, Wang X, Ge C, et al. Draft genome of spinach and transcriptome diversity of 120 Spinacia accessions. Nat Commun. 2017;8. doi:10.1038/ncomms15275

12. Yagi M, Kosugi $\mathrm{S}$, Hirakawa H, Ohmiya A, Tanase $\mathrm{K}$, Harada $\mathrm{T}$, et al. Sequence Analysis of the Genome of Carnation (Dianthus caryophyllus L.). DNA Res Int J Rapid Publ Rep Genes Genomes. 2014;21: 231-241. doi:10.1093/dnares/dst053

13. Lightfoot DJ, Jarvis DE, Ramaraj T, Lee R, Jellen EN, Maughan PJ. Single-molecule sequencing and Hi-C-based proximity-guided assembly of amaranth (Amaranthus hypochondriacus) chromosomes provide insights into genome evolution. BMC Biol. 2017;15: 74. doi:10.1186/s12915-017-0412-4

14. Jarvis DE, Ho YS, Lightfoot DJ, Schmöckel SM, Li B, Borm TJA, et al. The genome of Chenopodium quinoa. Nature. 2017;542: 307-312. doi:10.1038/nature21370

15. Copetti D, Búrquez A, Bustamante E, Charboneau JLM, Childs KL, Eguiarte LE, et al. Extensive gene tree discordance and hemiplasy shaped the genomes of North American columnar cacti. Proc Natl Acad Sci. 2017;114: 12003-12008. doi:10.1073/pnas.1706367114 
16. Shimada S, Takahashi K, Sato Y, Sakuta M. Dihydroflavonol 4-reductasecDNA from nonAnthocyanin-Producing Species in the Caryophyllales. Plant Cell Physiol. 2004;45: 1290-1298. doi:10.1093/pcp/pch156

17. Shimada S, Inoue YT, Sakuta M. Anthocyanidin synthase in non-anthocyanin-producing Caryophyllales species. Plant J. 2005;44: 950-959. doi:10.1111/j.1365-313X.2005.02574.x

18. Wickett NJ, Mirarab S, Nguyen N, Warnow T, Carpenter E, Matasci N, et al. Phylotranscriptomic analysis of the origin and early diversification of land plants. Proc Natl Acad Sci U S A. 2014;111: E4859-E4868. doi:10.1073/pnas.1323926111

19. Bolger AM, Lohse M, Usadel B. Trimmomatic: a flexible trimmer for Illumina sequence data. Bioinforma Oxf Engl. 2014;30: 2114-2120. doi:10.1093/bioinformatics/btu170

20. Pucker B, Holtgräwe D, Sörensen TR, Stracke R, Viehöver P, Weisshaar B. A De Novo Genome Sequence Assembly of the Arabidopsis thaliana Accession Niederzenz-1 Displays Presence/Absence Variation and Strong Synteny. PLOS ONE. 2016;11: e0164321. doi:10.1371/journal.pone.0164321

21. Andrews S. FastQC A Quality Control tool for High Throughput Sequence Data [Internet]. 2010 [cited 14 Dec 2017]. Available: https://www.bioinformatics.babraham.ac.uk/projects/fastqc/

22. Marçais G, Kingsford C. A fast, lock-free approach for efficient parallel counting of occurrences of k-mers. Bioinformatics. 2011;27: 764-770. doi:10.1093/bioinformatics/btr011

23. Vurture GW, Sedlazeck FJ, Nattestad M, Underwood CJ, Fang H, Gurtowski J, et al. GenomeScope: fast reference-free genome profiling from short reads. Bioinformatics. 2017;33: 2202-2204. doi:10.1093/bioinformatics/btx153

24. Luo R, Liu B, Xie Y, Li Z, Huang W, Yuan J, et al. SOAPdenovo2: an empirically improved memoryefficient short-read de novo assembler. GigaScience. 2012;1: 18. doi:10.1186/2047-217X-1-18

25. Cha S, Bird DM. Optimizing k-mer size using a variant grid search to enhance de novo genome assembly. Bioinformation. 2016;12: 36-40. doi:10.6026/97320630012036

26. Haak M, Vinke S, Keller W, Droste J, Rückert C, Kalinowski J, et al. High Quality de Novo Transcriptome Assembly of Croton tiglium. Front Mol Biosci. 2018;5. doi:https://doi.org/10.3389/fmolb.2018.00062

27. Keller O, Kollmar M, Stanke M, Waack S. A novel hybrid gene prediction method employing protein multiple sequence alignments. Bioinforma Oxf Engl. 2011;27: 757-763. doi:10.1093/bioinformatics/btr010

28. Simão FA, Waterhouse RM, loannidis $P$, Kriventseva EV, Zdobnov EM. BUSCO: assessing genome assembly and annotation completeness with single-copy orthologs. Bioinforma Oxf Engl. 2015;31: 3210-3212. doi:10.1093/bioinformatics/btv351

29. Li H. Aligning sequence reads, clone sequences and assembly contigs with BWA-MEM. ArXiv13033997 Q-Bio. 2013; Available: http://arxiv.org/abs/1303.3997

30. Hunt M, Kikuchi T, Sanders M, Newbold C, Berriman M, Otto TD. REAPR: a universal tool for genome assembly evaluation. Genome Biol. 2013;14: R47. doi:10.1186/gb-2013-14-5-r47 
31. Altschul SF, Gish W, Miller W, Myers EW, Lipman DJ. Basic local alignment search tool. J Mol Biol. 1990;215: 403-410. doi:10.1016/S0022-2836(05)80360-2

32. Holtgräwe D, Rosleff Sörensen T, Parol-Kryger R, Pucker B, Kleinbölting N, Viehöver P, et al. Low coverage re-sequencing in sugar beet for anchoring assembly sequences to genomic positions [Internet]. 2017. Available: https://jbrowse.cebitec.uni-bielefeld.de/RefBeet1.5/

33. Stadermann KB, Weisshaar B, Holtgräwe D. SMRT sequencing only de novo assembly of the sugar beet (Beta vulgaris) chloroplast genome. BMC Bioinformatics. 2015;16. doi:10.1186/s12859-015-0726-6

34. Kubo T, Nishizawa S, Sugawara A, Itchoda N, Estiati A, Mikami T. The complete nucleotide sequence of the mitochondrial genome of sugar beet (Beta vulgaris L.) reveals a novel gene for tRNACys(GCA). Nucleic Acids Res. 2000;28: 2571-2576.

35. Dobin A, Davis CA, Schlesinger F, Drenkow J, Zaleski C, Jha S, et al. STAR: ultrafast universal RNA-seq aligner. Bioinforma Oxf Engl. 2013;29: 15-21. doi:10.1093/bioinformatics/bts635

36. Smit A, Hubley R, Green P. RepeatMasker Frequently Open-4.0 [Internet]. 2015. Available: http://www.repeatmasker.org/

37. Green P. Consed--A Finishing Package [Internet]. [cited 11 Feb 2019]. Available: http://www.phrap.org/consed/consed.html\#howToGet

38. Kent WJ. BLAT-The BLAST-Like Alignment Tool. Genome Res. 2002;12: 656-664. doi:10.1101/gr.229202

39. Pucker B, Holtgräwe D, Weisshaar B. Consideration of non-canonical splice sites improves gene prediction on the Arabidopsis thaliana Niederzenz-1 genome sequence. BMC Res Notes. 2017;10. doi:https://doi.org/10.1186/s13104-017-2985-y

40. Minoche AE, Dohm JC, Schneider J, Holtgräwe D, Viehöver P, Montfort M, et al. Exploiting single-molecule transcript sequencing for eukaryotic gene prediction. Genome Biol. 2015;16: 184. doi:10.1186/s13059-015-0729-7

41. Nawrocki EP, Eddy SR. Infernal 1.1: 100-fold faster RNA homology searches. Bioinformatics. 2013;29: 2933-2935. doi:10.1093/bioinformatics/btt509

42. Kalvari I, Argasinska J, Quinones-Olvera N, Nawrocki EP, Rivas E, Eddy SR, et al. Rfam 13.0: shifting to a genome-centric resource for non-coding RNA families. Nucleic Acids Res. 2018;46: D335-D342. doi:10.1093/nar/gkx1038

43. Cheng C-Y, Krishnakumar V, Chan AP, Thibaud-Nissen F, Schobel S, Town CD. Araport11: a complete reannotation of the Arabidopsis thaliana reference genome. Plant J. 2017;89: 789804. doi:10.1111/tpj.13415

44. Jones P, Binns D, Chang H-Y, Fraser M, Li W, McAnulla C, et al. InterProScan 5: genome-scale protein function classification. Bioinformatics. 2014;30: 1236-1240. doi:10.1093/bioinformatics/btu031

45. Stanke M, Keller O, Gunduz I, Hayes A, Waack S, Morgenstern B. AUGUSTUS: ab initio prediction of alternative transcripts. Nucleic Acids Res. 2006;34: W435-W439. doi:10.1093/nar/gkl200 
AdditionalFile 9. Gene prediction statistics. Different gene prediction approaches were performed

46. Sterck L, Rombauts $S$, Vandepoele K, Rouzé $P$, Van de Peer $Y$. How many genes are there in plants (... and why are they there)? Curr Opin Plant Biol. 2007;10: 199-203. doi:10.1016/j.pbi.2007.01.004

47. Pucker B, Brockington SF. Genome-wide analyses supported by RNA-Seq reveal non-canonical splice sites in plant genomes. BMC Genomics. 2018;19: 980. doi:10.1186/s12864-018-5360-z

\section{Supporting Information}

AdditionalFile 1. Sequencing result overview.

AdditionalFile 2. Genome size estimation results. Genome size estimations with GenomeScope [23] are listed for various k-mer sizes. Two different read sets of $M$. australis were used for the genome size estimation (1=ERR2401802, 2=ERR2401614) to check the reproducibility.

AdditionalFile 3. Evaluation of assembly attempts of $K$. caespitosa.

AdditionalFile 4. Evaluation of assembly attempts of $M$. australis.

AdditionalFile 5. Evaluation of assembly attempts of $P$. exiguum.

\section{AdditionalFile 6. Detailed list of assembly parameters.}

Additionalfile 7. Gene prediction hint sources. These RNA-Seq read data sets and transcriptome assemblies were incorporated in the gene annotation process as hints.

AdditionalFile 8. Assembly attempt evaluation results. Statistics of raw assemblies were calculated to identify the best parameter settings. Since k-mer size was previously reported as the most important parameter, extensive optimization was performed. In addition, different settings for insert sizes were evaluated for $P$. exiguum (phe001-phe006). Parameter optimization for $M$. australis was performed on a subset of all reads due to availability.

during the optimization process. Results of these predictions include $a b$ initio gene prediction and 
bioRxiv preprint doi: https://doi.org/10.1101/646133; this version posted February 11,2020 . The copyright holder for this preprint (which was not certified by peer review) is the author/funder, who has granted bioRxiv a license to display the preprint in perpetuity. It is made available under aCC-BY 4.0 International license.

434 hint-based approaches. RNA-Seq reads and coding sequences derived from previous transcriptome

435 assemblies are two incorporated hint types. In addition, we assessed the impact of repeat masking

436 prior to gene prediction.

437 


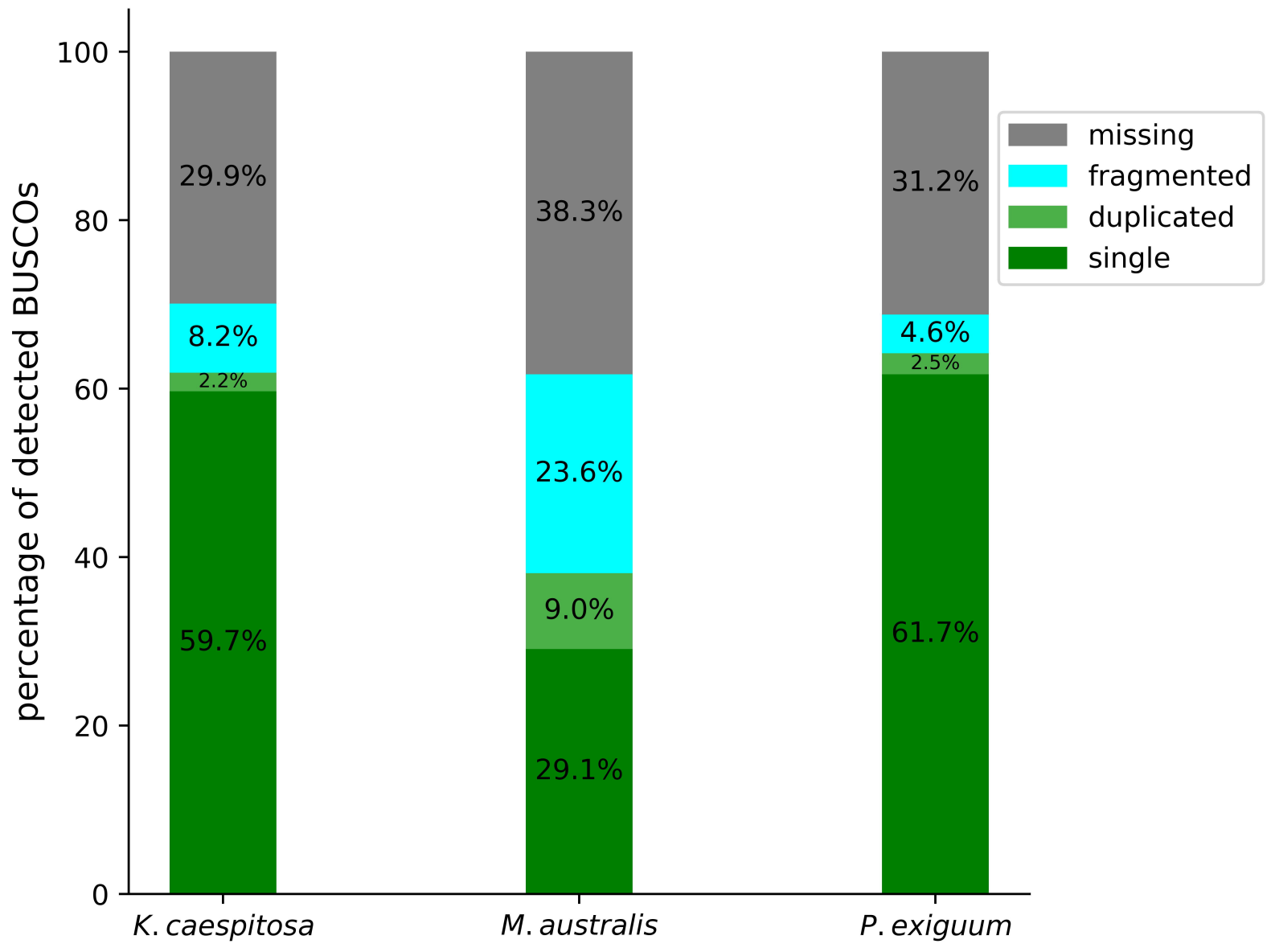




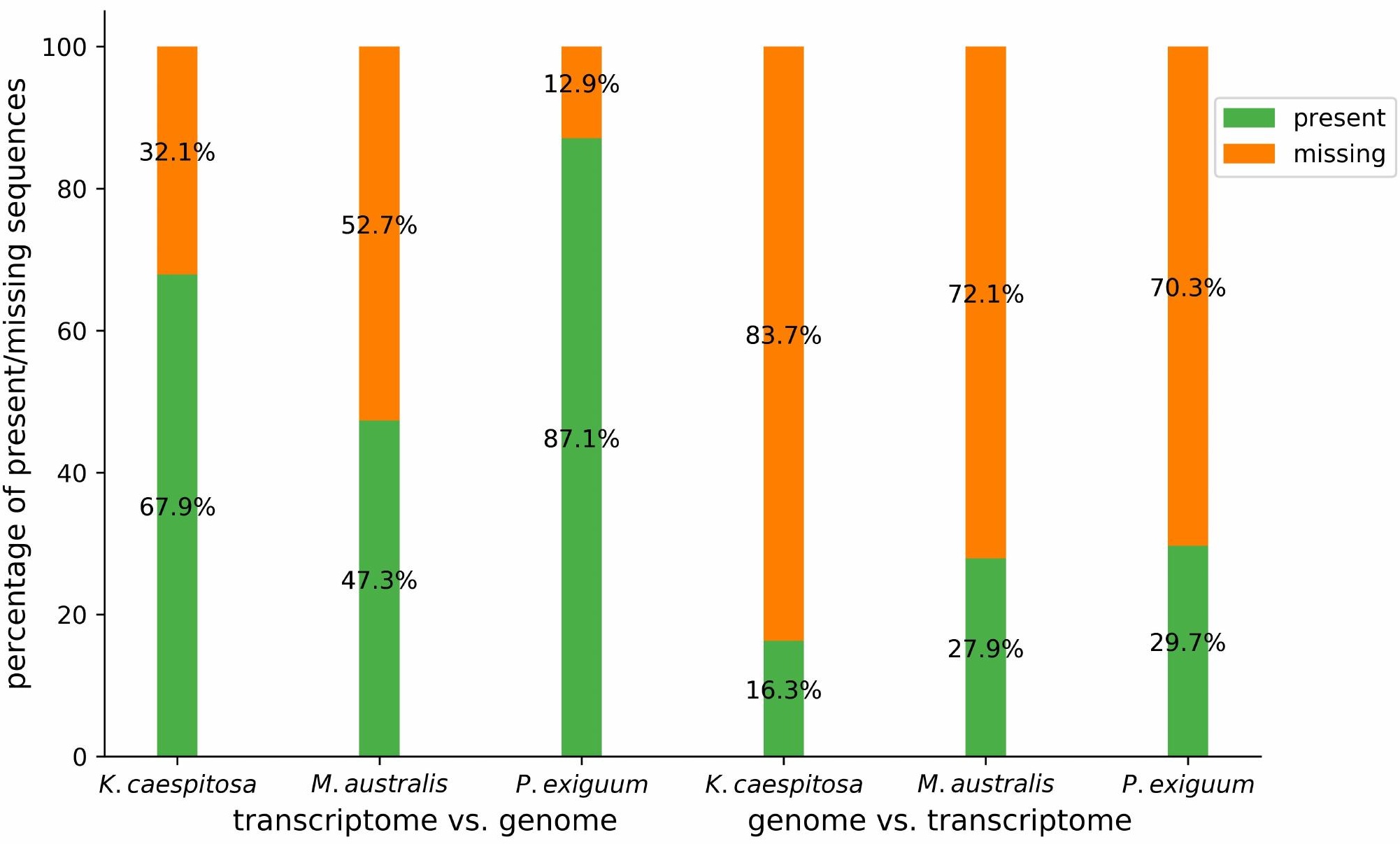

